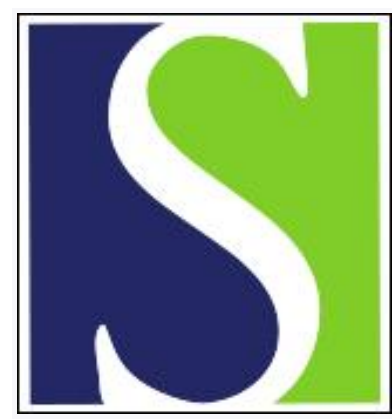

Scand J Work Environ Health 2008;34(4):260-266

https://doi.org/10.5271/sjweh.1225

Published online: 22 May 2008, Issue date: Aug 2008

Sick leaves in four factories-do characteristics of employees and work conditions explain differences in sickness absence between workplaces?

by Virtanen P, Siukola A, Luukkaala T, Savinainen M, Arola H, Nygård $\mathrm{C}-\mathrm{H}$, Kivimäki M, Helenius $\mathrm{H}$, Vahtera J

Affiliation: P Virtanen, Tampere School of Public Health, FI-37014 University of Tampere, Finland. pekka.j.virtanen@uta.fi

Refers to the following text of the Journal: 2001;27(6):365-372

The following articles refer to this text: 2008;34(4):235-238;

2009;35(4):241-243; 2009;35(6):429-436; 2010;36(5):394-403; 2010;36(6):515-516; 2011;37(3):213-218

Key terms: blue-collar worker; employee; factory; Finland; food industry; multilevel analysis; musculoskeletal diagnosis; physical work conditions; psychosocial work conditions; sick leave; sickness absence; work conditions; workplaces

This article in PubMed: www.ncbi.nlm.nih.gov/pubmed/18604460 


\title{
Sick leaves in four factories-do characteristics of employees and work conditions explain differences in sickness absence between workplaces?
}

\author{
by Pekka Virtanen, MO, ${ }^{1}$ Anna Siukola, MSc, ${ }^{1}$ Tiina Luukkaala, MSc, ${ }^{2}$ Minna Savinainen, PhD,${ }^{1}$ Heikki \\ Arola, MD, ${ }^{3}$ Clas-Håkan Nygård, PhD, ${ }^{1}$ Mika Kivimäki, PhD, ${ }^{4}$ Hans Helenius, MSc, ${ }^{5}$ Jussi Vahtera, MD ${ }^{6}$
}

\begin{abstract}
Virtanen P, Siukola A, Luukkaala T, Savinainen M, Arola H, Nygård C-H, Kivimäki M, Helenius H, Vahtera J. Sick leaves in four factories-do characteristics of employees and work conditions explain differences in sickness absence between workplaces? Scand J Work Environ Health 2008;34(4):260-266.
\end{abstract}

\begin{abstract}
Objectives The study explored whether differences in sickness absence between four factories of a food industry company were explained by common determinants of sickness absence, such as employee health, sociodemographic characteristics, and physical and psychosocial work conditions.

Methods Survey responses of 582 employees were linked to the records of short-term (1-3 days) and long-term ( $>3$ days) absence, as well as to records of absences due to musculoskeletal diagnoses. Multilevel models were applied in assessing the between-factory absence differences.

Results Compared with the levels in the factory with the lowest sickness absence, in one factory the levels of short-term [rate ratio (RR) 1.72], long-term (RR 1.96), and musculoskeletal (RR 2.93) absence were significantly higher. Another factory also had higher levels of long-term and musculoskeletal absence (RR 2.17 and 2.52, respectively). Adjustment for the background factors explained $35 \%$ of the difference in short-term absence, $3-9 \%$ of the differences in long-term absence, and 18-12\% of the differences in musculoskeletal absence, but the between-factory differences were still highly significant.

Conclusions This study showed large differences in sickness absence between factories that were only partly explained by common determinants. Moreover, economic factors and formal control were unlikely explanatory factors, as the study was conducted within a single company. These results justify further research on local absence practices and cultures, including those of health service organizations and professionals.
\end{abstract}

Key terms blue-collar worker; Finland; food industry; multilevel analysis; musculoskeletal diagnosis; physical work conditions; psychosocial work conditions.

The starting point of this study was the difference observed in sickness absence between factories of a food industry company. We sought explanations for these differences in the characteristics of the employees, the jobs, and the organizations.

Sickness absence involves a complex set of reasons, ranging from national sickness insurance and labor legislation to individual health problems. It indicates, by definition, temporary work disability due to the illness of an employee. In addition to temporary illness, chronic diseases and poor perceived health are reflected in higher absence rates (1-4), and high long-term absence predicts permanent work disability and death (5-7). Earlier studies also show that past absence is an independent predictor of future absence (8).

Sickness absence is more common among women $(1,9)$ and depends on age, short periods being more common at a younger age and long periods occurring

1 Tampere School of Public Health, University of Tampere, Tampere, Finland.

2 Tampere University Hospital, Tampere, Finland.

3 Suomen terveystalo, Helsinki, Finland.

4 University College London, London, United Kingdom.

5 University of Turku, Turku, Finland.

6 Finnish Institute of Occupational Health, Turku, Finland.

Correspondence to: Dr P Virtanen, Tampere School of Public Health, FI-37014 University of Tampere, Finland. [E-mail: pekka.j.virtanen@uta.fi] 
more often among older employees (1). The association between high socioeconomic status and a low absence rate has repeatedly been shown irrespective of the indicator of socioeconomic status $(4,10,11)$.

Physical workload and a physically demanding job increase the risk of sickness absence $(12,13)$, as do adverse physical work conditions (2). A large body of research suggests associations between psychosocial work conditions and sickness absence. For example, low decision authority $(11,14)$ and a low level of job autonomy and low job complexity (15) have been found to be associated with an increased risk of absence. There is also evidence that favorable changes in job control, job demands, and social support reduce the risk (16). However, studies finding no association between these work characteristics and sickness absence have also been reported $(2,8)$, and some studies suggest that the associations may be dependent on gender $(1,11,15)$. The low absence level of fixed-term employees (17) has been interpreted as a tendency towards "sickness presence", or working while ill.

Although employee health and sociodemographic and job characteristics have been shown to be important determinants of sickness absence, it remains unclear whether these three factors explain a substantial share of the variation in sickness absence between workplaces. It has been hypothesized that a considerable part of this variation remains to be attributed to practices resulting from the moral and cultural communication that takes place and develops in localities and work communities. Our earlier studies suggest that such "sickness absence habitus" is a possible explanation for differences in sickness absence between workplaces beyond the common determinants $(18,19)$. However, the register data used in these studies did not allow a detailed analysis of employee health and work conditions. Moreover, the studies were limited to public-sector employees and therefore left unanswered the question of whether a corresponding phenomenon occurs among blue-collar workers in private-sector industries.

This study of a food industry company with multiple factories was based on data from a sickness absence register and a questionnaire survey concerning employee health and job characteristics. The responses to the questionnaires were collated with the register to examine the extent to which differences in sickness absence across factories were explained by the major reviewed determinants.

\section{Study population and methods}

The study was carried out in a leading food processing company in Finland. From the personnel register of the company, we obtained data on the age, gender, contract (length, permanent or fixed-term, and monthly or hourly wages), workplace (four factories and administration center), and sickness absence of all employees employed in 2002-2003. In addition to the dates when the sick leaves started and stopped, the place where certificates were issued, as well as the diagnosis, according to the 10th revision of the International Classification of Diseases (ICD-10), were recorded to be used by occupational health care in statistics about the health of personnel. Data on all sick leaves from 1 January 2002 to 31 December 2003 were obtained from this register. The duration of job contract represented the "time at risk", from which the time absent from work for reasons other than sickness was subtracted. Sickness absence was expressed as the rates per person-year for short (1-3 days) and long (over 3 days) periods and for periods due to musculoskeletal diagnoses.

Questionnaire surveys were implemented in the company during the first half of 2003. The employees were asked to assess their health "compared with that of others of the same age" on a scale ranging from 0 (poor) to 10 (excellent). A similar response scale was used in questions concerning current work ability as compared with that at its best $(0=$ totally unable to work, $10=$ work ability at its best), and the amount of stress $(0=$ not at all, $10=$ very much stress).

Ergonomics was addressed with a question about repetitive movements and a question about poor work postures, giving the choice on a 5-point Likert scale. In addition, physical strain was elicited using the scale from 6 to 20 (20). Environmental exposure was assessed with questions about any inconvenience caused by noise, draft, heat, cold, poor quality of internal air, and poor or blinding lighting. The replies to the six questions with a 5 -point Likert scale were summed into a single variable ranging from 6 to 30 . These variables were entered into the multivariate models as scores.

The psychosocial work conditions studied were job control, team spirit, quality of leadership, and possibilities to exert influence. Job control was measured with an index including 18 questions on the content of the work, independence, opportunities to participate, and role clarity (16). The sum of the response scores was expressed as a percentage of maximum job control. The other scales (21) used comprised six proposition items on the spirit and functioning of the team (sample item: "My colleagues discuss improvements to work and/or the work environment"), six items on incentive and participative elements of leadership (sample item: "My manager pays attention to my suggestions and wishes"), and five propositions about influence (sample item: "The organization allows its employees an opportunity to set their own goals"). The responses were given on 5-point Likert scales from 1 = "totally disagree/very probably 
not" to 5 = "totally agree/very probably". The results were presented as mean scores, also ranging from 1.00 to 5.00. The Cronbach's alphas of these three measures were $0.86,0.88$, and 0.79 , respectively.

The questionnaire was distributed to all of the employees of the company during the spring of 2003. The closed reply envelopes were collected at the workplaces and sent to the researchers. The forms were not addressed to individual employees; thus no reminders could be sent. The respondents were requested to provide written consent for their survey data to be collated with their records, including sickness absence, in the personnel register. The survey yielded 1120 respondents, of whom $873(78 \%)$ gave their name and consent. In this study, we included the respondents who consented, worked in one of the four target factories, received hourly wages (indicating explicitly manual worker status), and had an employment contract of at least 6 months either in 2002 or 2003, a total of 582 participants. The study was approved by the ethics committee of the Pirkanmaa University Hospital District.

The chi-square test, a univariate analysis of variance, and the Kruskall-Wallis test were used to study the between-factory differences of the background variables. The differences in absence between the factories were determined with rates (absences per person-year), and their statistical significance was analyzed according to multilevel models. According to the prerequisites for multilevel analyses, our dataset included persons (employees) nested within factories. In the multilevel analysis, the factory was included in the models as a random effect. We first tested the significance of the factory-level variance component by comparing the -2 log likelihood statistics of a model treating factory as a random factor to a corresponding model without factory. A significantly better fit in the random-effect model, compared with the model without factory, was observed in all of the analyses $(\mathrm{P}<0.001)$, and therefore the use of multilevel models were justified in which factory was a random effect. Because sickness absence is a rare event and constitutes count data, the distribution of this discrete variable was modeled with the Poisson distribution assumption. The results were expressed by estimating the average absence levels in the factories using the best linear unbiased predictions for factory. First, we calculated the unadjusted predictions. We adjusted the models for age and gender and then for health indicators and physical and psychosocial work conditions. Finally, we defined the rate ratios and calculated the proportion of the between-factory difference explained by the background variables calculated with the formula (unadjusted RR - adjusted RR) / (unadjusted RR - 1). For all of the analyses we used the SAS 9.1 program package (SAS Inc, Cary, NC, USA), and they were conducted using the nlmixed procedure.

\section{Results}

\section{Sample characteristics}

Table 1 presents the characteristics of the survey respondents by factory. The sickness absence rates for short and long periods, as well as for the sick leaves due to musculoskeletal morbidity, were lower in factories $\mathrm{A}$ and $\mathrm{D}$ than in factories $\mathrm{B}$ and $\mathrm{C}$. There were more female respondents in factories $\mathrm{A}, \mathrm{B}$, and $\mathrm{C}$ and more male respondents in factory D. Age differences were nonsignificant. According to the survey-based variables, poor health was the least common in factory $\mathrm{A}$, and poor work ability the least common in factory D. The employees of factory $\mathrm{C}$ were the least stressed. The work in factory A was physically demanding, and there were high environmental exposures, but the ergonomic quality of the jobs was better than in factories B and C. Regarding the psychosocial conditions, factory D stood out in terms of incentive leadership, factory B in terms of poor opportunities to exert influence, and factory A in terms of good job control.

\section{Nonresponse analysis}

For the nonresponse analysis, we identified all of the employees who, according to the personnel register, had been contracted for at least 6 months during 2003. Of the total of 1995 employees, 1443 were hourly paid workers (249 in factory A, 367 in factory B, 625 in factory C, and 200 in factory D). A comparison of the respondents with all hourly paid workers showed that the differences in absence levels were small in factories A, B and C, whereas, in factory $\mathrm{D}$, the respondents' absence rate was relatively low (figure 1). The age distribution of the respondents (table 1) was similar for all of the eligible workers (the proportion of employees over 40 years of age being $54 \%, 43 \%, 43 \%$, and $37 \%$, respectively). The gender distributions were also similar in factories $\mathrm{A}$ and $\mathrm{B}$, in factory $\mathrm{C}$ the proportion of women was somewhat lower among the respondents (68\% versus $78 \%)$, and in factory D this proportion was somewhat higher $(30 \%$ versus $18 \%$ ).

\section{Differences in sickness absence between factories}

The between-factory variation in sickness absence proved to be highly significant (table 2). Introduction of the explanatory factors into the multilevel analyses somewhat narrowed the range of best linear unbiased predictions for both short-term absence and absence due to musculoskeletal morbidity, while the differences remained nearly unchanged for long-term absence. In a comparison with factory $\mathrm{A}$, adjustment for age, gender, and all indicators of health, physical work conditions 
Table 1. Descriptive statistics of the respondents (N) and their work conditions by factory.

\begin{tabular}{|c|c|c|c|c|c|c|c|c|c|}
\hline & \multicolumn{2}{|c|}{ Factory $A(N=76)$} & \multicolumn{2}{|c|}{ Factory $B(N=144)$} & \multicolumn{2}{|c|}{ Factory C (N=322) } & \multicolumn{2}{|c|}{ Factory $D(N=40)$} & \multirow[t]{2}{*}{ P-value } \\
\hline & $\%$ & Mean & $\%$ & Mean & $\%$ & Mean & $\%$ & Mean & \\
\hline \multicolumn{10}{|l|}{ Employment contract } \\
\hline Fixed-term & 10 & . & 7 & . & 4 & . & 4 & . & \\
\hline Permanent & 90 & $\cdot$ & 93 & $\cdot$ & 96 & . & 96 & $\cdot$ & \\
\hline \multicolumn{10}{|c|}{ Sickness absence spells/person-year } \\
\hline Short $(<4$ days $)$ & . & 1.76 & . & 2.45 & . & 3.20 & . & 1.95 & \\
\hline Long (over 3 days) & . & 0.92 & . & 1.97 & . & 1.89 & . & 0.88 & \\
\hline Musculoskeletal & . & 0.46 & . & 1.29 & . & 1.47 & . & 0.57 & \\
\hline Gender & & & & & & & & & $<0.001$ \\
\hline Men & 32 & . & 30 & . & 22 & . & 70 & . & \\
\hline Women & 68 & . & 70 & . & 78 & . & 30 & . & \\
\hline Age & & & & & & & & & 0.217 \\
\hline$\leq 29$ years & 21 & . & 24 & . & 26 & . & 30 & . & \\
\hline 30-39 years & 26 & . & 31 & . & 25 & . & 30 & . & \\
\hline 40-49 years & 22 & . & 29 & . & 25 & . & 33 & . & \\
\hline$\geq 50$ years & 30 & . & 17 & $\cdot$ & 24 & . & 8 & . & \\
\hline \multicolumn{10}{|l|}{ Health ${ }^{a}$} \\
\hline Poor general health & 33 & . & 39 & . & 40 & . & 35 & . & 0.851 \\
\hline Impaired work ability & 19 & . & 22 & . & 22 & . & 10 & . & 0.578 \\
\hline High stress & 33 & . & 39 & . & 28 & . & 39 & . & 0.633 \\
\hline \multicolumn{10}{|l|}{ Physical work conditions } \\
\hline High physical strain ${ }^{\mathrm{b}}$ & 50 & . & 46 & . & 34 & . & 45 & . & 0.013 \\
\hline Repetitive movements ${ }^{c}$ & 40 & . & 55 & . & 45 & . & 48 & . & 0.772 \\
\hline Poor work postures ${ }^{c}$ & 31 & . & 52 & . & 46 & . & 25 & . & 0.043 \\
\hline High environmental exposure ${ }^{a}$ & 45 & . & 39 & $\cdot$ & 37 & $\cdot$ & 18 & $\cdot$ & 0.030 \\
\hline \multicolumn{10}{|l|}{ Psychosocial work conditions } \\
\hline Team spirit ${ }^{d}$ & . & 3.26 & . & 3.09 & . & 3.22 & . & 3.31 & $<0.001$ \\
\hline Incentive leadership ${ }^{d}$ & . & 3.14 & . & 3.15 & . & 3.13 & . & 3.27 & $<0.001$ \\
\hline Influence possibilities ${ }^{d}$ & $\cdot$ & 3.35 & . & 2.98 & $\cdot$ & 3.15 & . & 3.32 & $<0.001$ \\
\hline Job controle & 52 & $\cdot$ & 45 & · & 45 & $\cdot$ & 48 & · & 0.001 \\
\hline
\end{tabular}

a Percentage of workers belonging to the lowest or poorest tertile.

${ }^{\mathrm{b}}$ Borg, 1970 (20).

c Percentage of replies 4 or 5 on a Likert scale of 1-5.

d Mean of the score $1-5$.

e Index (percentage of the maximum).

and psychosocial work conditions together reduced the 1.72-fold rate of short sick leaves in factory $\mathrm{C}$ by $35 \%$, but the difference still remained statistically significant. Corresponding reductions of the 2.17-fold and 1.96-fold rates of long sick leaves in factories $\mathrm{B}$ and $\mathrm{C}$, respectively, were only $3 \%$ and $9 \%$, respectively. The estimates for musculoskeletal sick leaves in factories $\mathrm{B}$ and $\mathrm{C}$ were also higher than average, and adjustments reduced them by $18 \%$ and $12 \%$, respectively.

\section{Correlates of sickness absence}

Finally, we examined whether the traditional determinants of sickness absence were confirmed in this sample. As expected, sickness absence was associated with gender, age, perceived health, stress, work ability, and work conditions (physical strain, work postures, job control, and employment contract). For example, the rate ratio (RR) (Poisson regression analysis with full adjustment) for long-term absence was 1.38 [95\% confidence interval
(95\% CI) 1.22-1.56] for the women when they were compared with the men, and 1.24 (95\% CI 1.05-1.46) for employees reporting high physical strain when they were compared with those reporting low strain.

\section{Discussion}

The starting point for this study was a considerably lower sickness absence level in one of the four factories of a food industry company. Traditional determinants of sickness absence as factors contributing to this difference were sought using questionnaire data. We found that a comprehensive set of factors related to the workers, to the characteristics of the work, and to the work conditions was associated with sickness absence, but it explained only part of the difference in short periods of absence and hardly part of the difference in long sickness absences. 
Short-term (1-3 days) absence

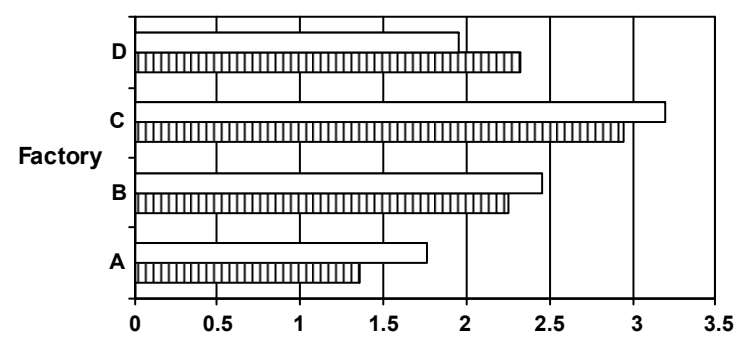

Long-term (>3 days) absence

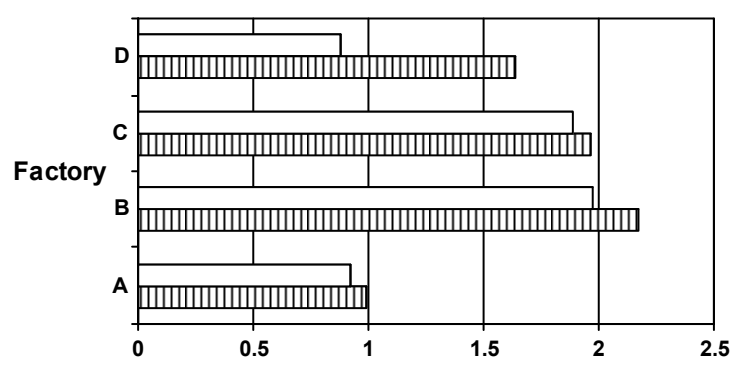

Absence due to musculoskeletal morbidity

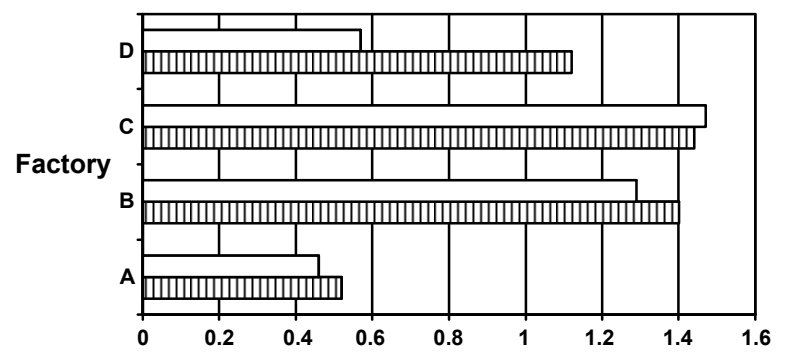

Figure 1. Absences per person-year for all workers in the four factories and for the workers participating in the study. ( $\square=$ participants, $\square=$ all workers)

The number of employees included in the analysis may seem low, but it is, in part, explained by our inclusion criteria. The original response rate of 56\% (1120 of 1995 workers) is acceptable, and the actual rate was even higher because our definition of the eligible population represented an overestimate. Exactly at the time of the survey, the company had about 1800 employees (instead of 1995). The distribution of the questionnaires by the administration may also have caused suspicions and increased the tendency towards nonresponse and towards refusal to consent. Indeed, only for factory D should the figures be interpreted with caution because the response rate remained modest, and, as shown in figure 1, in this factory the absence figures of the respondents were clearly lower than the figures for all of the workers. The following discussion concentrates therefore on the findings of factories A, B, and C.

The failure of traditional determinants to capture a considerable part of locality-related differences in sick-
Table 2. Estimates of the average absence per person-year [best linear unbiased estimates and their 95\% confidence intervals $(95 \% \mathrm{Cl})$ ] for short (1-3 days) and long (over 3 days) sickness absences and for absences due to a musculoskeletal diagnosis among blue-collar workers in four factories. P-values refer to the significance of the factory-level component.

\begin{tabular}{|c|c|c|c|c|c|c|}
\hline \multirow{3}{*}{$\begin{array}{l}\text { Sickness } \\
\text { absence }\end{array}$} & \multicolumn{6}{|c|}{ Adjustments } \\
\hline & \multicolumn{2}{|c|}{ Unadjusted } & \multicolumn{2}{|c|}{ Gender and age } & \multicolumn{2}{|c|}{ Fulla } \\
\hline & Mean & $95 \% \mathrm{Cl}$ & Mean & $95 \% \mathrm{Cl}$ & Mean & $95 \% \mathrm{Cl}$ \\
\hline \multicolumn{7}{|l|}{ Short } \\
\hline Factory A & 0.79 & $0.53-1.16$ & 0.82 & $0.58-1.16$ & 0.86 & $0.64-1.16$ \\
\hline Factory B & 1.08 & $0.74-1.56$ & 1.06 & $0.76-1.48$ & 1.02 & $0.77-1.35$ \\
\hline Factory C & 1.36 & $0.95-1.97$ & 1.31 & $0.95-1.81$ & 1.26 & $0.96-1.66$ \\
\hline Factory D & \multicolumn{2}{|c|}{$P<0.001$} & \multicolumn{2}{|c|}{$P<0.001$} & \multicolumn{2}{|c|}{$P<0.001$} \\
\hline \multicolumn{7}{|l|}{ Long } \\
\hline Factory A & 0.71 & $0.36-1.37$ & 0.70 & $0.39-1.29$ & 0.69 & $0.38-1.22$ \\
\hline Factory B & 1.54 & $0.81-2.93$ & 1.50 & $0.84-2.68$ & 1.48 & $0.84-2.53$ \\
\hline Factory C & 1.39 & $0.73-2.63$ & 1.32 & $0.74-2.34$ & 1.29 & $0.75-2.24$ \\
\hline Factory D & \multicolumn{2}{|c|}{$P<0.001$} & \multicolumn{2}{|c|}{$P<0.001$} & \multicolumn{2}{|c|}{$P<0.001$} \\
\hline \multicolumn{7}{|c|}{ Musculoskeletal } \\
\hline Factory A & 0.58 & $0.26-1.31$ & 0.58 & $0.27-1.25$ & 0.59 & $0.29-1.23$ \\
\hline Factory B & 1.46 & $0.67-3.18$ & 1.42 & $0.68-2.99$ & 1.33 & $0.67-2.63$ \\
\hline Factory C & 1.70 & $0.78-3.69$ & 1.63 & $0.78-3.40$ & 1.59 & $0.81-3.13$ \\
\hline Factory D & \multicolumn{2}{|c|}{$P<0.001$} & \multicolumn{2}{|c|}{$\begin{array}{l}0.76 \quad 0.34-1.70 \\
P<0.001\end{array}$} & \multicolumn{2}{|c|}{$\begin{array}{c}0.81 \quad 0.38-1.71 \\
P<0.001\end{array}$} \\
\hline
\end{tabular}

a In addition to gender and age, health-related variables, physical work conditions, and psychosocial work conditions (variables, see table 1).

ness absence is consistent with the hypothesis of sickness absence habitus. We use this concept, originating from the sociologist Pierre Bourdieu (22), to refer to the "generative scheme ... in society, or 'knowing without knowing' how to act properly .... . In the case of sickness absence, too, habitus is channeled along prescribed paths into individuals' and groups' practices: hot to be ill is a matter of distinction and discourse about the dominant way of life, style and taste [p 1226-1227]" (18).

Short-term absence is assumed to be related to minor or incipient health problems, whereas long-term absence is typically thought to reflect unavoidable work disability related to serious impairment $(3,7)$. From this point of view, health and other traditional determinants would be expected to fail to explain locality and workplace-related differences in short-term rather than in long-term sickness absence. We found the opposite, the finding suggesting that unmeasured explanatory factors, or habitus, might contribute, in particular, to long-term absence certified by physicians.

In the studied company a certificate was required for every day of sick leave. The division into short-term and long-term absence describes roughly the division between nurse-certified and physician-certified absence. Health care professionals' practices and attitudes towards sickness absence may vary (23), and the decision 
to take sick leave may also depend on access to health services (24). However, it is improbable that the local health service system would prove to be the major reason for the low absence level in factory A. On the other hand, health care also contributes to the sickness absence practices of the community it serves. Thus there would be grounds for studying the services available in the respective localities.

The different composition of cause-specific sick leaves may confound observed differences in the overall absence figures between workplaces. For musculoskeletal diagnoses, the between-factory difference was somewhat more pronounced than for all absence. Differences in ergonomic and physical work conditions between the factories partly explained the variation, but the rate ratios for factories $\mathrm{B}$ and $\mathrm{C}$ remained relatively high. Thus the case of musculoskeletal diagnoses supports rather than contradicts the view that the reasons for the between-factory differences lie largely beyond traditionally known risk factors.

Any factory-A-specific bias or defect in the data is improbable, as the guidelines for recording data on sick leaves were consistent throughout the company. Moreover, the respondents proved to be a representative sample of blue-collar workers as regards age and gender. Their absence rate also corresponded with that of the total personnel in factories A, B, and C.

Sickness absence is strongly associated with the economy. Studies indicate that macroeconomic fluctuations, such as recessions and upswings and associated variations in the unemployment rate, are reflected in sickness absence statistics $(25,26)$. The economic conditions of an enterprise and associated solutions regarding personnel policy also affect sickness absence. Fear of redundancy may reduce absences (27), but in the longer run downsizing may increase them, in particular if no corresponding reduction in production, or workload, takes place, as is typical in public-sector services (28). Outsourcing or the use of agency workers may "outsource" sickness absence or reduce the absence statistics visible in the records of the main employer. However, the company representatives reported no differences in these factors between the factories.

One feature known to affect absence is the size of the workplace. The rate tends to be low in small workplaces (29). In line with this knowledge, we found the highest total rate (4.90) in the biggest factory $(\mathrm{C})$ and the second highest rate (4.42) in the second biggest factory (B), but the rate in factory A (2.34) was clearly lower than in the smallest factory D (3.96). Thus the factory size logics would estimate the "proper" rate for factory A at somewhere between 3.96 and 4.42 .

The amount of absence also depends on sick pay (30-32). The factories compared in this study belonged to the same company, and therefore the economic factors were similar. Moreover, the union-level agreements were applied similarly in the factories (ie, all of the employees were paid full salary during sick leave, and the requirements for medical certificates were uniform). Thus the observed differences in absence cannot be attributed to factory-specific economic conditions or remuneration.

Measuring work conditions with a questionnaire is open to subjectivity bias, but there is no reason to assume that such biases or measurement imprecision were factory specific. Thus the observed differences between factories are unlikely to be attributable to measurement error. However, the range of work conditions studied was limited. Features not captured in this study, such as operational procedures, safety culture, organizational justice, or even the personalities of key persons, may have exerted influences on sickness absence and therefore explained the residual differences between the workplaces. A further possibility is that these differences arise from workplace-specific sickness absence cultures, which are relatively independent of the aspects of psychosocial work conditions that are usually studied. If the roots of such a culture are to be revealed, it is essential to study everyday absence practices in relation to the community in and around the workplace.

Finally, we return to "sickness absence habitus". The observed large unexplained between-factory variation in sickness absence is pending further study with respect to factors contributing to factory-specific sick-leave practices. The results provide a basis for qualitative data collection in the factories with focus on the social contexts and cultures surrounding sickness absence. The locality-level perspective should also be adopted. Although a factory may be more independent than, for example, the municipality $(18,19)$, it is part and parcel of the local community, and we can ask how far the social, economic, and political power relationships of geographically distinct societies are constructed collectively into distinctive styles of assuming the sick role.

\section{Acknowledgments}

This study was supported by the Finnish Work Environment Fund, grant 102308. MK and JV were supported by the Academy of Finland, grants 105195, 117604, and 124271.

\section{References}

1. Blank N, Diderichsen F. Short-term and long-term sick-leave in Sweden: relationships with social circumstances, working conditions and gender. Scand J Soc Med. 1995;23:265-72. 
2. Hanebuth D, Meinel M, Fischer JE. Health-related quality of life, psychosocial work conditions, and absenteeism in an industrial sample of blue- and white-collar employees: a comparison of potential predictors. J Occup Environ Med. 2006;48:28-37.

3. Marmot M, Feeney A, Shipley M, North F, Syme SL. Sickness absence as a measure of health status and functioning: from the UK Whitehall II study. J Epidemiol Community Health. 1995;49:124-30.

4. North F, Syme SL, Feeney A, Head J, Shipley MJ, Marmot MG. Explaining socioeconomic differences in sickness absence: The Whitehall II study. BMJ. 1993;306:361-6.

5. Kivimäki M, Head J, Ferrie JE, Shipley MJ, Vahtera J, Marmot MG. Sickness absence as a global measure of health: evidence from mortality in the Whitehall II prospective cohort study. BMJ. 2003;327:364.

6. Kivimäki M, Ferrie JE, Hagberg J, Head J, Westerlund H, Vahtera $\mathbf{J}$, et al. Diagnosis-specific sick leave as a risk marker for disability pension in a Swedish population. J Epidemiol Community Health. 2007;61:915-20.

7. Vahtera J, Pentti J, Kivimäki M. Sickness absence as a predictor of mortality among male and female employees. J Epidemiol Community Health. 2004;58:321-6.

8. Smulders PGW, Nijhuis FJN. The job demands-job control model and absence behaviour: results of a 3-year longitudinal study. Work Stress. 1999;13:115-31.

9. Mastekaasa A, Olsen KM. Gender, absenteeism, and job characteristics: a fixed effects approach. Work Occup. 1998;25:195-228.

10. Alexanderson K, Leijon M, Akerlind I, Rydh H, Bjurulf P. Epidemiology of sickness absence in a Swedish county in 1985, 1986 and 1987: a three year longitudinal study with focus on gender, age and occupation. Scand J Soc Med. 1994;22:27-34.

11. Kivimäki M, Vahtera J, Thomson L, Griffiths A, Cox T, Pentti J. Psychosocial factors predicting employee sickness absence during economic decline. J Appl Psychol. 1997;82:858-72.

12. Boedeker W. Associations between workload and diseases rarely occurring in sickness absence data. J Occup Environ Med. 2001;43:1081-8.

13. Vingård E, Lindberg $\mathrm{P}$, Josephson $\mathrm{M}$, Voss $\mathrm{M}$, Heijbel B, Alfredsson L, et al. Long-term sick-listing among women in the public sector and its associations with age, social situation, lifestyle, and work factors: a three-year follow-up study. Scand J Public Health. 2005;33(5):370-5.

14. Christensen KB, Nielsen ML, Rugulies R, Smith-Hansen L, Kristensen TS. Workplace levels of psychosocial factors as prospective predictors of registered sickness absence. J Occup Environ Med. 2005;47:933-40.

15. Väänänen $A$, Toppinen-Tanner $S$, Kalimo R, Mutanen $P$, Vahtera J, Peiró JM. Job characteristics, physical and psychological symptoms, and social support as antecedents of sickness absence among men and women in the private industrial sector. Soc Sci Med. 2003;57:807-24.

16. Vahtera J, Kivimäki M, Pentti J, Theorell T. Effect of change in the psychosocial work environment on sickness absence: a seven year follow up of initially healthy employees. J Epidemiol Community Health. 2000;54:484-93.

17. Virtanen M, Kivimäki M, Elovainio M, Vahtera J, Cooper CL. Contingent employment, health and sickness absence. Scand J Work Environ Health. 2001;27(6):365-72.

18. Virtanen P, Nakari R, Ahonen H, Vahtera J, Pentti J. Locality and habitus: the origins of sickness absence practices. Soc Sci Med. 2000;50:27-39.

19. Virtanen P, Vahtera J, Nakari R, Pentti J, Kivimäki M. Economy and job contract as contexts of sickness absence practices: revisiting locality and habitus. Soc Sci Med. 2004;58:1219-29.

20. Borg C. Perceived exertion as an indicator of somatic stress. Scand J Rehabil Med. 1970;2:92-8.

21. Ruohotie P. Ammatillinen kasvu työelämässä [Professional growth at work]. Hämeenlinna (Finland): Tampereen yliopiston Hämeenlinnan opettajankoulutuslaitos; 1993. Ammattikasvatussarja 8.

22. Bourdieu P. Outline of a theory of practice. Cambridge (United Kingdom): Cambridge University Press; 1977.

23. Arrelov BE, Borgquist L, Svardsudd KF. Influence of local structural factors on physicians' sick-listing practice: a population-based study. Eur J Public Health. 2005;15:470-4.

24. Lofland JH, Frick KD. Workplace absenteeism and aspects of access to health care for individuals with migraine headache. Headache. 2006;46:563-76.

25. Salminen J. Talouslama ja sairauslomat [Economic depression and sick leave]. Suomen Lääkärilehti. 2003;58:21-4.

26. Virtanen M, Kivimäki M, Elovainio M, Virtanen P, Vahtera J. Local economy and sickness absence: prospective cohort study. J Epidemiol Community Health. 2005;59:973-8.

27. Virtanen P. An epidemic of 'good health' at the work-place. Sociol Health Illness. 1994;16:394-401.

28. Vahtera J, Kivimäki M, Pentti J. Effect of organisational downsizing on health of employees. Lancet. 1997;350:1124-8.

29. Voss M, Floderus B, Diderichsen F. Physical, psychosocial, and organisational factors relative to sickness absence: a study based on Sweden Post. Occup Environ Med. 2001;58:17884.

30. Engström LG, Eriksen T. Can differences in benefit levels explain duration and outcome of sickness absence? Disabil Rehabil. 2002;24:713-8.

31. Henrekson M, Persson M. The effects on sick leave of changes in the sickness insurance system. J Labor Econ. 2004;22:87113.

32. Voss M, Floderus B, Diderichsen F. Changes in sickness absenteeism following the introduction of a qualifying day for sickness benefit-findings from Sweden Post. Scand J Public Health. 2001;29:166-74.

Received for publication: 22 March 2007 